\title{
Identification of key pathways and candidate genes in pancreatic ductal adenocarcinoma using bioinformatics analysis
}

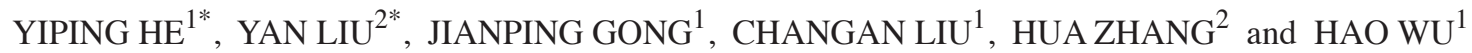 \\ ${ }^{1}$ Department of Hepatobiliary Surgery, The Second Affiliated Hospital of Chongqing Medical University, \\ Chongqing 400010; ${ }^{2}$ Department of Gastroenterology, The Fifth People's Hospital of Chengdu, \\ Chengdu, Sichuan 611130, P.R. China
}

Received June 20, 2018; Accepted February 6, 2019

DOI: $10.3892 / \mathrm{ol} .2019 .10041$

\begin{abstract}
Pancreatic ductal adenocarcinoma (PDAC) is a malignant tumor with a high degree of malignancy that is difficult to diagnose and treat. The present study integrated PDAC cohort profile datasets to identify key candidate genes and pathways involved in the pathogenesis of the disease. The expression profiles of GSE28735 included 45 PDCA and matching pairs of adjacent non-tumor tissue. Differentially expressed genes (DEGs) were sorted and candidate genes and pathway enrichment were analyzed. A DEG-associated protein-protein interaction (PPI) network was constructed. A total of 424 DEGs were identified in PDAC, including 159 upregulated genes and 265 downregulated genes. Gene Ontology analysis results indicated that upregulated DEGs were significantly enriched in biological process, molecular function and cellular component categories. Kyoto Encyclopedia of Genes and Genomes pathway analysis demonstrated that the upregulated DEGs were enriched in 'pancreatic secretion', 'protein digestion' and 'absorption'. Downregulated DEGs were enriched in 'ECM-receptor interaction', 'focal adhesion' and 'PI3K/AKT' signaling pathways. The PPI network revealed that these genes were involved in significant pathways, including 'ECM organization' signaling pathways (Hippo signaling pathway, TGF- $\beta$ signaling pathway, Hedgehog signaling pathway and Wnt signaling pathway), 'serine-type peptidase activity' signaling pathway (PI3K-Akt signaling pathway, TNF- $\alpha$ signaling pathway and Wnt signaling pathway) and 'extracellular region' signaling
\end{abstract}

Correspondence to: Professor Hao Wu, Department of Hepatobiliary Surgery, The Second Affiliated Hospital of Chongqing Medical University, 76 Linjiang Road, Chongqing 400010, P.R. China

E-mail:whwh1hero@163.com

${ }^{*}$ Contributed equally

Key words: pancreatic ductal adenocarcinoma, bioinformatics analysis, differentially expressed genes pathways (RTP signaling pathway, G protein-coupled receptor signaling pathway and RAS-RAF-MAPK signaling pathway). The identification of these candidate genes and pathways sheds light on the etiology and molecular mechanisms of PDAC and may guide the development of novel therapies for pancreatic cancer.

\section{Introduction}

Pancreatic cancer has a poor prognosis, with a median survival time of 3-6 month and a 5-year survival rate of less than 5\% (1-3). The most common type of pancreatic cancer is pancreatic ductal adenocarcinoma (PDAC), accounting for $\sim 90 \%$ of pancreatic cancer cases (4). Although numerous studies have focused on the pathogenesis and progression of pancreatic cancer, the etiology and molecular mechanisms of pancreatic cancer remain unclear $(5,6)$. Previous scientific studies have demonstrated that the occurrence and progression of pancreatic cancer involve the interaction of several factors, including gene mutations and environmental conditions $(7,8)$. Thus far, there remains a lack of information regarding the molecular mechanisms that cause the development and progression of pancreatic cancer that would allow for improved precision therapies. Therefore, understanding the molecular mechanisms of pancreatic cancer can provide an effective basis for early prevention, diagnosis and treatment.

The advent of the gene chip and high-throughput gene analysis platforms allows for the rapid detection of gene expression in a microarray, which is particularly suitable for screening differentially expressed genes (DEGs) (9). With the widespread application of gene chip technology in cancer research, a large amount of genetic data has been produced and stored in public gene databases. Classification, integration and analysis of these data can provide valuable insights and evidence for cancer research. In the past few years, numerous gene chip expression profiles have been used to study the pathogenesis and development of PDAC and hundreds of DEGs have been identified (10). However, due to differences in sample size and limitations of the studies, no reliable biomarkers were identified. The combination of gene chip and biological information analysis technology can be used to monitor the expression of DEGs in the development and 
progression of PDAC and to elucidate the signaling pathways involved, potentially revealing targets which can be modulated to treat PDAC (11).

In the present study, the original GSE28735 data set (12) was downloaded from the Gene Expression Omnibus (GEO) database (13). The dataset contained the gene expression profiles of 45 matching pairs of pancreatic tumor and adjacent non-tumor tissues from 45 patients with PDAC. DEGs were detected by comparing the gene expression profiles between tumor tissues and paracancerous tissues in patients with PDAC. Subsequently, the DEGs were filtered using the Morpheus website (https://software.broadinstitute.org/morpheus/) with data processing standard. Then, the DEGs were screened using the Gene-Spring software (version 11.5; Agilent Technologies, Inc., Santa Clara, CA, USA), followed by Gene Ontology (GO); (www.geneontology.org) and pathway enrichment analysis. In addition, a protein-protein interaction (PPI) network was established and three significant modules were analyzed. The analysis of the biological pathways underlying the development of PDAC may provide information for its diagnosis, prognosis and treatment.

\section{Materials and methods}

Microarray data. The gene expression profiles of the GSE28735 dataset were downloaded from the GEO database. The GPL6244 [HuGene-1_0-st] Affymetrix Human Gene 1.0 ST Array platform (Affymetrix; Thermo Fisher Scientific, Inc., Waltham, MA, USA) was used. The GSE28735 dataset contained 90 samples, including 45 PDAC tumor samples and 45 matching pairs of adjacent non-tumor tissue samples.

Identification of DEGs in GSE28735. The raw expression data files include TXT files (Affymetrix platform) used for analysis by processing using the Morpheus website. Data were categorized into two groups with similar expression patterns in PDAC tumor samples and matching pairs of adjacent non-tumor tissue samples. A t-test was used to identify the DEGs and $l \log _{2}$ fold changel $\geq 1$ and $\mathrm{P}<0.05$ were considered statistically significant.

Gene ontology and pathway enrichment analysis of DEGs. GO analysis was used to annotate genes and classify up and downregulated DEGs. GO terms are divided into three main categories: Biological process (BP), cellular component (CC) and molecular function (MF). The Kyoto Encyclopedia of Genes and Genomes (KEGG; www.kegg.jp) website is an online database which contains defined and associated gene sets and their pathways. The Database for Annotation, Visualization and Integrated Discovery (DAVID; david. ncifcrf.gov) allows analysis of gene lists and provides biological information regarding genes. To analyze the upregulated and downregulated genes in DEGs, GO and KEGG pathway analysis were used in the DAVID database. $\mathrm{P}<0.05$ was considered to indicate a statistically significant difference.

Integration of PPI network. The Search Tool for the Retrieval of Interacting Genes (STRING; www.string-db. org) was used to evaluate the PPI information. The PPI network served to identify the key genes and Cytoscape software (version 3.51; www.cytoscape.org) was used to draw the network diagram. The topology of the PPI network was analyzed and the extent of the expression of each gene was calculated. $\mathrm{P}<0.05$ was considered to indicate a statistically significant difference.

\section{Results}

Identification of DEGs in pancreatic cancer. A total of 45 PDAC tumor samples and 45 matching pairs of adjacent non-tumor tissue samples were analyzed. A total of 424 DEGs were identified from GSE28735, including 159 upregulated and 265 downregulated genes (Table I). The heat map of DEG expression, presenting the top 50 upregulated and 50 downregulated genes was constructed using the web-based tool Morpheus (Fig. 1).

GO term and pathway enrichment analyses. To further elucidate the function of the selected genes, the online software DAVID was used to perform DEG GO analysis. As aforementioned, GO analysis results classify DEG functions and pathways into three functional groups: $\mathrm{BP}, \mathrm{CC}$ and $\mathrm{MF}$. For BP, the upregulated DEGs were enriched in 'digestion', 'lipid digestion' and 'proteolysis', while the downregulated DEGs were enriched in 'ECM organization', 'extracellular structure organization' and 'cell adhesion' (Tables II and III). For CC, the upregulated DEGs were enriched in the 'extracellular region', and the downregulated DEGs were enriched in 'extracellular region' and 'ECM' (Tables II and III). For MF, the upregulated DEGs were enriched in 'serine-type peptidase activity', 'serine hydrolase activity' and 'peptidase activity', and the downregulated DEGs were enriched in 'ECM structural constituent', 'integrin binding' and 'cell adhesion molecule binding' (Tables II and III).

KEGG pathway analysis in pancreatic cancer. KEGG pathway analysis was used to analyze the most significantly enriched pathways of the upregulated DEGs and downregulated DEGs. The upregulated DEGs were enriched in 'pancreatic secretion', 'protein digestion and absorption' and 'fat digestion and absorption' (Table IV). The downregulated DEGs were enriched in 'ECM-receptor interaction', 'focal adhesion' and 'PI3K/Akt signaling' pathways (Table IV).

PPI and modular analysis in pancreatic cancer. Using the STRING online database and Cytoscape software analysis, a total of 386 DEGs (143 upregulated and 243 downregulated genes) of the 424 commonly altered DEGs were filtered into the DEGs PPI network complex, including 424 nodes and 1090 edges (Fig. 2A). The 10 nodes with the highest degree were cystic fibrosis transmembrane conductance regulator (CFTR), SLC7A2 (solute carrier family 7 member 2), C-C motif chemokine ligand 18 (CCL18), pyruvate dehydrogenase kinase 4(PDK4), BAI1 associated protein 2 like 1 (BAIAP2L1), integrin subunit $\alpha 3$ (ITGA3), carboxypeptidase A1 (CPA1), G protein-coupled receptor class C group 5 member A (GPRC5A), serine/threonine/tyrosine kinase 1 (STYK1), and ST6 N-acetylgalactosaminide $\alpha$-2, 6 -sialyltransferase 1 (ST6GALNAC1). Among the upregulated DEGs, a total of 
Table I. A total of 424 DEGs were identified from the GSE28735 dataset, including 159 upregulated genes and 265 downregulated genes in PDAC tissues, compared to adjacent non-tumor tissue samples.

Differential expression

Upregulated

Downregulated

DEGs, differentially expressed genes; PDAC, pancreatic ductal adenocarcinoma.
Gene symbol

EPB41L4B, FAM129A, SLC1A2, KLKB1, ALDH1A1, PAH, CHGA, CHST9, SEMA6A, SERPINA5, KIF1A, CHRDL1, SLC16A10, CLU, MIR27B, PRKAR2B, FAM3B, ADHFE1, LONRF2, DPT, CHRM3, SLC3A1, ABAT, PPY, BNIP3, NUCB2, GPHA2, ATRNL1, ESRRG, ABCA8, FAM150B, ONECUT1, PRSS3P2, OR4D5, CXCL12, IL22RA1, TSPAN7, F8, GCG, ADGRV1, SV2B, UGT2B11, SPINK1, PROX1, ANGPTL1, UNC79, AMY2B, MCOLN3, AQP12B, FAM159B, FOSB, BTG2, SLC43A1, FLRT2, GSTA1, AQP12B, C5, SCG3, CCDC141, DPP10,PKHD1, PRSS3, C2CD4B, MT1G, HOMER2, GRB14,LYVE1, BACE1, SLC39A5, CD36, RGN, SYCN, GC, EPHX2, REG3G, DCDC2, GUCA1C, SST, PCSK1, PDZK1P1, BEX1, PRSS2, LIFR, GRPR, SLC30A8, MIR217, LMO3, ANKRD62, CTNND2, PM20D1, CFTR, GNMT, TFPI2, SLC17A4, PAK3, GSTA2, AMY2B, G6PC2, TTN, CELP, SLC4A4, PRSS3P2, C6, TTR, QP8, SLC7A2, KCNJ16, PDK4, OR8D4, REG3A, FABP4, NRCAM, NRG4, PAIP2B, GATM, FGL1, ACADL, ADH1B, TRHDE, RBPJL, SCGN, REG1A, PRSS1, CPB1, SLC16A12, ANPEP, TMED6, KLK1, RO1B, F11, CTRB2, AOX1, NR5A2, KIAA1324, CELA3B, EGF, CPA1, PDIA2, REG1CP, EG1B, PNLIP, CTRB1, CTRL, CELA3A, CELA2B, CELA2A, PLA2G1B, SERPINI2, CLPS, ERP27, FAM24B, ALB, CPA2, CEL, GP2, CTRC, IAPP, PNLIPRP2, PNLIPRP1

CEACAM5, SLC6A14, LAMC2, GALNT5, TSPAN1, CTSE, POSTN, CEACAM6, ANXA10, LAMB3, ITGA2, TMPRSS4, FN1, COL11A1, SERPINB5, DPCR1, AGR2, CLDN18, ITGB6, KRT19, GABRP, CST1, VSIG1, SULF1, TFF1, COL17A1, SLC2A1, PLAC8, CEMIP, SLPI, CP, AHNAK2, MMP12, COL12A1, TMC5, VCAN, MUC17, KRT7, ANLN, INHBA, TRIM31, LIPH, CDH3, TRIM31, SCEL, NOX4, THBS2, EGLN3, C13, ADGRF1, MBOAT2, ANTXR1, TCN1, ANKRD22, COL10A1, CXCL5, XYD3, KRT17, BCAS1, ITGA3, SDR16C5, EDIL3, APOL1, UGT1A3, COL1A1, MMP11, FERMT1, FAP, ANXA8L1, CDH11, COL1A2, MET, FNDC1, FBXO32, COMP, NQO1, ACSL5, MLPH, NPR3, ANXA8L1, MIA-RAB4B, COL8A1, GCNT3, IGFL2,ADAMTS12, TNS4, CAPG, TRIM29, TSPAN8, CYP2C18, TRIM31, TMEM45B, MATN3, COL5A2, PLAU, PADI1, ITGA11, COL3A1, CCL20, IGFP5, LAMA3, HK2, IFI27, MYOF, PLAT, FER1L6, KRT6C, ECT2, LY75, MMP14, TOP2A, DNRA, LEF1, CENPF, TNFAIP6, ITGB4, PLEK2, CEACAM1, LAMP5, TMC7, NPR3, OLR1, SERPINB3, ANO1, DHRS9, SLC6A6, MICAL2, MUC16, ARNTL2, PTPRR, KYNU, NRP2, S100A14, CD109, BAIAP2L1, AFAP1-AS1, LOXL2, FGD6, CST2, IFI44L, S100P, MMP1, COL6A3, SL44A4, ERO1A, ASPM, BGN, DKK1, STYK1, MMP7, RUNX2, NT5E, TGM2, HEPH, KRT17, GPX2, OSBPL3, LMO7, GPRC5A, EPHA4, DCP1, GF2BP3, S100A16, PXDN, MKI67, EFNA5, KRT17, MELK, ADAM9, SLC22A3, MST1R, ACTA2, FF2, LCN2, PLPP4, ADAM28, MXRA5, DPYSL3, TGFBI, XDH, CCL18, OAS1, ABHD17C, RHBDL2, HIST1H3H, MUC1, INPP4B, AEBP1, MMP9, MTMR11, FOXQ1, ENO2, OCIAD2, DLGAP5, HPGD, TPX2, PLA2R1, SRPX2, LRRN1, SLCO1B3, SEMA3C, IL1RAP, SYTL2, FER1L4, DSG2, SULF2, HOXB5, MFP5, IL2RG, SULT1B1, CORIN, SLC9A2, GJB2, ADAM12, PLS1, AK4, ATP2C2, GREM1, ETV1, LTBP1, OAS2, ASAP2, SGIP1, PGM2L1, DDX60, DGKH, KCNN4, MALL, P4HA1, ANXA3, TSK, EPYC, NRP2, FUT3, ADAMTS6, KRT6A, IL1R2, DCBLD2, NMU, EFNB2, ST6GALNAC1, ANGPT2, FCGR3B, KIF23, FBN1, PKM, SEMA7A, TRIM16, RTKN2, SLC26A9, NTM, PCDH7, RAI14, SULT1C2, ESM1, AREG, DSG3, GPX8, MACC1, CTHRC1, HIST1H3I, SCNN1A, SLC16A3

143 DEGs were filtered into the DEG PPI network complex including 143 nodes and 263 edges (Fig. 2B), which were mainly associated with 'digestion', 'serine-type peptidase activity' and the 'extracellular region' (Table V). Among the downregulated DEGs, a total of 143 DEGs were filtered into the DEGs PPI network complex including 243 nodes and 


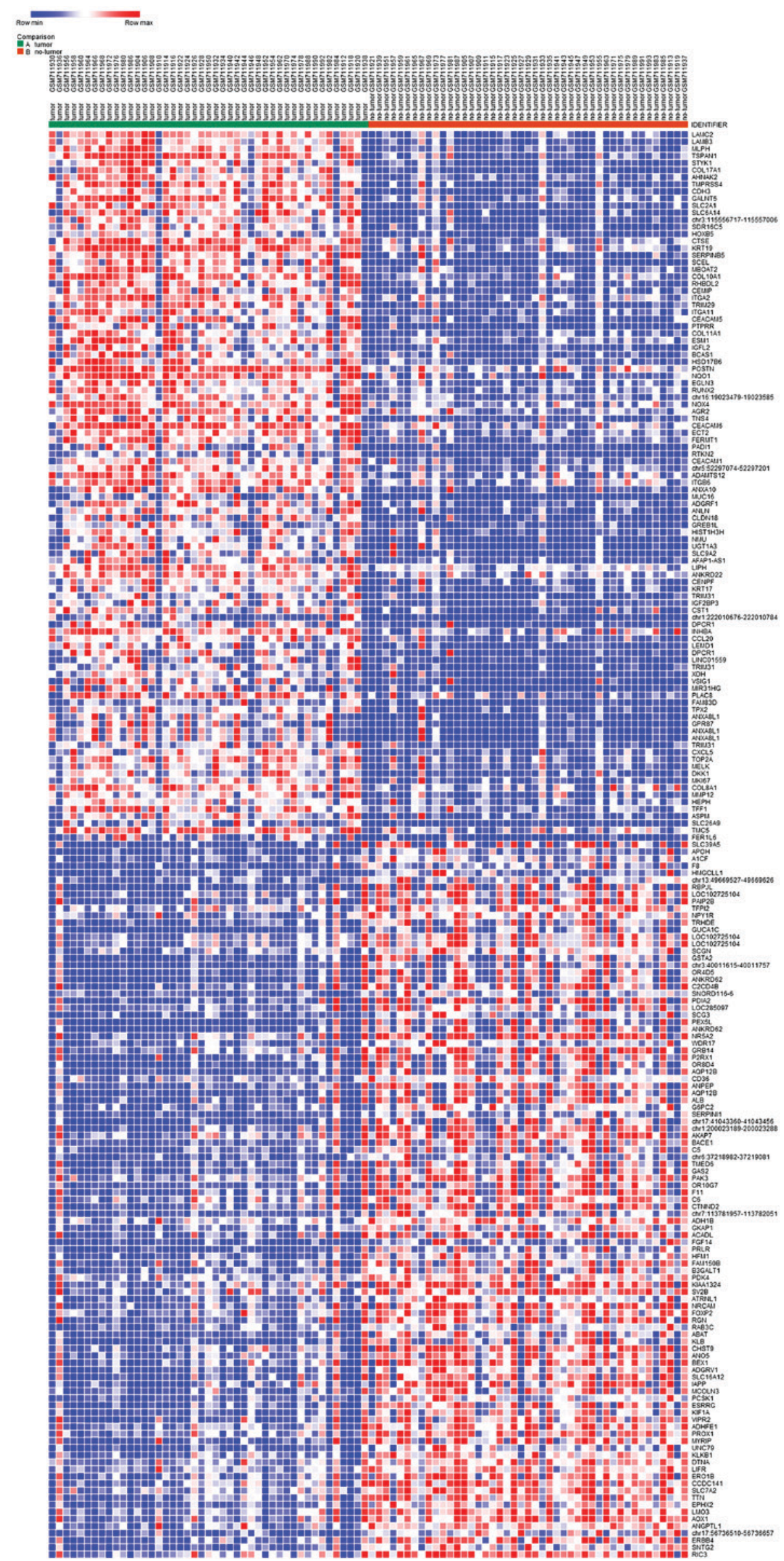

Figure 1. Heat map of the top 100 differentially expressed genes in PDAC. The heat map presents 50 upregulated genes (red) and 50 downregulated genes (blue). DEGs, differentially expressed genes; PDAC, pancreatic ductal adenocarcinoma. 
Table II. GO analysis of upregulated DEGs associated with PDAC.

\begin{tabular}{|c|c|c|c|c|}
\hline Category & Term & Gene function & Count & P-value \\
\hline BP & GO:0007586 & Digestion & 18 & $3.31 \times 10^{-14}$ \\
\hline $\mathrm{BP}$ & GO:0044241 & Lipid digestion & 6 & $9.18 \times 10^{-7}$ \\
\hline $\mathrm{BP}$ & GO:0006508 & Proteolysis & 35 & $1.08 \times 10^{-6}$ \\
\hline $\mathrm{BP}$ & GO:0006766 & Vitamin metabolic process & 9 & $1.57 \times 10^{-5}$ \\
\hline $\mathrm{BP}$ & GO:0009235 & Cobalamin metabolic process & 5 & $2.56 \times 10^{-5}$ \\
\hline $\mathrm{BP}$ & GO:0015850 & Organic hydroxy compound transport & 10 & $4.03 \times 10^{-5}$ \\
\hline $\mathrm{BP}$ & GO:0006767 & Water-soluble vitamin metabolic process & 7 & $9.77 \times 10^{-5}$ \\
\hline BP & GO:0006629 & Lipid metabolic process & 26 & $1.11 \times 10^{-4}$ \\
\hline $\mathrm{BP}$ & GO:0046903 & Secretion & 23 & $1.23 \times 10^{-4}$ \\
\hline BP & GO:0032940 & Secretion by cell & 21 & $1.67 \times 10^{-4}$ \\
\hline $\mathrm{CC}$ & GO:0005576 & Extracellular region & 90 & $1.47 \times 10^{-17}$ \\
\hline $\mathrm{CC}$ & GO:0005615 & Extracellular space & 47 & $4.64 \times 10^{-15}$ \\
\hline $\mathrm{CC}$ & GO:0044421 & Extracellular region part & 78 & $8.75 \times 10^{-15}$ \\
\hline $\mathrm{CC}$ & GO:0031988 & Membrane-bounded vesicle & 64 & $6.61 \times 10^{-9}$ \\
\hline $\mathrm{CC}$ & GO:0070062 & Extracellular exosome & 52 & $1.46 \times 10^{-7}$ \\
\hline $\mathrm{CC}$ & GO:1903561 & Extracellular vesicle & 52 & $1.72 \times 10^{-7}$ \\
\hline $\mathrm{CC}$ & GO:0043230 & Extracellular organelle & 52 & $1.74 \times 10^{-7}$ \\
\hline $\mathrm{CC}$ & GO:0030141 & Secretory granule & 15 & $4.27 \times 10^{-6}$ \\
\hline $\mathrm{CC}$ & GO:0060205 & Cytoplasmic membrane-bounded vesicle lumen & 8 & $4.51 \times 10^{-5}$ \\
\hline $\mathrm{CC}$ & GO:0031983 & Vesicle lumen & 8 & $4.80 \times 10^{-5}$ \\
\hline $\mathrm{MF}$ & GO:0008236 & Serine-type peptidase activity & 18 & $2.13 \times 10^{-10}$ \\
\hline MF & GO:0017171 & Serine hydrolase activity & 18 & $2.51 \times 10^{-10}$ \\
\hline MF & GO:0008233 & Peptidase activity & 27 & $3.41 \times 10^{-10}$ \\
\hline $\mathrm{MF}$ & GO:0004252 & Serine-type endopeptidase activity & 17 & $4.20 \times 10^{-10}$ \\
\hline MF & GO:0070011 & Peptidase activity, acting on L-amino acid peptides & 26 & $8.65 \times 10^{-10}$ \\
\hline MF & GO:0004175 & Endopeptidase activity & 18 & $8.45 \times 10^{-7}$ \\
\hline MF & GO:0008238 & Exopeptidase activity & 9 & $4.27 \times 10^{-6}$ \\
\hline $\mathrm{MF}$ & GO:0008235 & Metalloexopeptidase activity & 6 & $1.98 \times 10^{-4}$ \\
\hline MF & GO:0004806 & Triglyceride lipase activity & 4 & $7.93 \times 10^{-4}$ \\
\hline $\mathrm{MF}$ & GO:0005179 & Hormone activity & 6 & $3.54 \times 10^{-3}$ \\
\hline
\end{tabular}

GO, gene ontology; DEGs, differentially expressed genes; PDAC, pancreatic ductal adenocarcinoma; BP, biological process; MF, molecular function; $\mathrm{CC}$, cellular component.

497 edges (Fig. 2C), which were mainly associated with 'ECM organization', 'ECM structural constituents' and the 'extracellular region’ (Table VI).

\section{Discussion}

The incidence of PDAC is increasing worldwide (14). The clinical signs and symptoms may be difficult to diagnose in the initial stages of the disease (7). Patients are often diagnosed at a late stage, when regional invasion or distant metastasis have occurred, resulting in a 5-year survival rate of $\sim 5 \%(15,16)$. An insight into the molecular mechanisms of PDAC would allow for earlier diagnosis and more effective treatment. The rapid development of gene chips and high-throughput sequencing can rapidly and accurately provide gene expression data for thousands of genes in the human genome. Previous studies have identified some of the genes and signaling pathways that serve a role in the development of pancreatic cancer from chip analysis $(17,18)$. In the present study, the chip data in the GSE28735 dataset was comprehensively analyzed and 424 common DEGs (159 upregulated and 265 downregulated) between PDAC and matching pairs of adjacent non-tumor tissue were identified using bioinformatics analysis.

GO analysis is an international standardized gene function classification system that provides the molecular function of genes involved in a variety of biological processes (19). In the current study, GO term analysis revealed that the upregulated genes were mainly involved in 'digestion', 'lipid digestion' and 'proteolysis', and downregulated DEGs were involved in 'extracellular matrix organization', 'extracellular structure 
Table III. GO analysis of downregulated DEGs associated with PDAC.

\begin{tabular}{|c|c|c|c|c|}
\hline Category & Term & Gene function & Count & P-value \\
\hline $\mathrm{BP}$ & GO:0030198 & Extracellular matrix organization & 42 & $2.61 \times 10^{-27}$ \\
\hline $\mathrm{BP}$ & GO:0043062 & Extracellular structure organization & 42 & $2.94 \times 10^{-27}$ \\
\hline $\mathrm{BP}$ & GO:0007155 & Cell adhesion & 67 & $2.12 \times 10^{-15}$ \\
\hline $\mathrm{BP}$ & GO:0022610 & Biological adhesion & 67 & $2.52 \times 10^{-15}$ \\
\hline $\mathrm{BP}$ & GO:0016477 & Cell migration & 53 & $3.81 \times 10^{-14}$ \\
\hline $\mathrm{BP}$ & GO:0030574 & Collagen catabolic process & 15 & $1.91 \times 10^{-13}$ \\
\hline $\mathrm{BP}$ & GO:0051674 & Localization of cell & 55 & $2.69 \times 10^{-13}$ \\
\hline $\mathrm{BP}$ & GO:0048870 & Cell motility & 55 & $2.69 \times 10^{-13}$ \\
\hline $\mathrm{BP}$ & GO:0044243 & Multicellular organism catabolic process & 15 & $8.59 \times 10^{-13}$ \\
\hline $\mathrm{BP}$ & GO:0006928 & Movement of cell or subcellular component & 64 & $1.18 \times 10^{-12}$ \\
\hline $\mathrm{CC}$ & GO:0005576 & Extracellular region & 148 & $4.33 \times 10^{-25}$ \\
\hline $\mathrm{CC}$ & GO:0044421 & Extracellular region part & 133 & $3.86 \times 10^{-24}$ \\
\hline $\mathrm{CC}$ & GO:0031012 & Extracellular matrix & 42 & $8.29 \times 10^{-18}$ \\
\hline $\mathrm{CC}$ & GO:0005578 & Proteinaceous extracellular matrix & 35 & $1.90 \times 10^{-17}$ \\
\hline $\mathrm{CC}$ & GO:0044420 & Extracellular matrix component & 19 & $1.21 \times 10^{-12}$ \\
\hline $\mathrm{CC}$ & GO:0005615 & Extracellular space & 59 & $6.91 \times 10^{-12}$ \\
\hline $\mathrm{CC}$ & GO:0070062 & Extracellular exosome & 84 & $6.11 \times 10^{-10}$ \\
\hline $\mathrm{CC}$ & GO:1903561 & Extracellular vesicle & 84 & $7.84 \times 10^{-10}$ \\
\hline $\mathrm{CC}$ & GO:0043230 & Extracellular organelle & 84 & $7.99 \times 10^{-10}$ \\
\hline $\mathrm{CC}$ & GO:0031988 & Membrane-bounded vesicle & 96 & $9.00 \times 10^{-9}$ \\
\hline MF & GO:0005201 & Extracellular matrix structural constituent & 13 & $1.27 \times 10^{-9}$ \\
\hline MF & GO:0005178 & Integrin binding & 14 & $2.41 \times 10^{-9}$ \\
\hline MF & GO:0050839 & Cell adhesion molecule binding & 25 & $2.52 \times 10^{-8}$ \\
\hline MF & GO:0019838 & Growth factor binding & 13 & $2.08 \times 10^{-7}$ \\
\hline MF & GO:0004222 & Metalloendopeptidase activity & 12 & $4.62 \times 10^{-7}$ \\
\hline MF & GO:0005539 & Glycosaminoglycan binding & 15 & $1.20 \times 10^{-6}$ \\
\hline MF & GO:0005509 & Calcium ion binding & 28 & $2.19 \times 10^{-6}$ \\
\hline MF & GO:0005518 & Collagen binding & 9 & $2.67 \times 10^{-6}$ \\
\hline MF & GO:0005102 & Receptor binding & 43 & $3.59 \times 10^{-6}$ \\
\hline MF & GO:0008237 & Metallopeptidase activity & 13 & $1.34 \times 10^{-5}$ \\
\hline
\end{tabular}

GO, gene ontology; DEGs, differentially expressed genes; PDAC, pancreatic ductal adenocarcinoma; BP, biological process; MF, molecular function; CC, cellular component.

organization' and 'cell adhesion'. The pancreas mainly secretes trypsin and pancreatic lipase and abnormalities in secretions can interfere with protein and lipid metabolism, leading to chronic pancreatitis which is one of the important contributing factors for pancreatic cancer (20). The stability of cell structure and cell adhesion is also a major factor in the formation of pancreatic cancer (21).

Furthermore, KEGG pathway analysis indicated that the upregulated DEGs were involved in pancreatic secretion pathways and protein and lipid digestion and absorption pathways. Existing studies revealed that metabolic change is considered one of the characteristics of cancer, especially the dysfunction of pancreatic secretion $(11,22)$. In pancreatic cancer, metabolic changes are prominent in protein and lipid digestion and absorption pathways (23). The downregulated DEGs were associated with 'ECM-receptor interaction', 'focal adhesion' and the 'PI3K-Akt signaling' pathways.

Previous studies indicated that pancreatic stellate cells, which can cause pancreatic fibrosis leading to pancreatic cancer, can produce and secrete ECM $(24,25)$. One of the components of ECM, hyaluronic acid, can combine with CD44 antigen and influence vascular epithelial-mesenchymal transition (EMT) as well as cancer cell resistance to chemotherapy (4). Furthermore, the main constitutive protein of ECM, collagen I, can promote the adhesion of pancreatic cancer cells through the proliferation and migration of integrin $\alpha 2 \beta 1$ (24). Collagen, fibronectin and laminin are also associated with chemoresistance in pancreatic cancer cells in vitro (26). Previous studies revealed that focal adhesions interact with the ECM and can promote EMT, 
Table IV. KEGG pathway analysis of DEGs associated with PDAC.

A, Upregulated

\begin{tabular}{|c|c|c|c|c|}
\hline Pathway & Name & Count & P-value & Genes \\
\hline hsa04972 & Pancreatic secretion & 19 & $4.1 \times 10^{-18}$ & $\begin{array}{l}\text { PNLIP, CELA3A, PNLIPRP1, CELA3B, PNLIPRP2, } \\
\text { PRSS1, CFTR, CEL, CHRM3, PRSS2, PRSS3, CPA2, } \\
\text { PLA2G1B, CELA2B, CELA2A, CPA1, CPB1, SLC4A4, } \\
\text { CTRL }\end{array}$ \\
\hline hsa04974 & $\begin{array}{l}\text { Protein digestion and } \\
\text { absorption }\end{array}$ & 13 & $1.9 \times 10^{-10}$ & $\begin{array}{l}\text { CELA3A, CELA3B, SLC16A10, PRSS2, PRSS3, PRSS1, } \\
\text { CPA2, CELA2B, CELA2A, CPA1, SLC3A1, CPB1, CTRL }\end{array}$ \\
\hline hsa04975 & $\begin{array}{l}\text { Fat digestion and } \\
\text { absorption }\end{array}$ & 7 & $4.1 \times 10^{-6}$ & $\begin{array}{l}\text { PNLIP, CEL, CLPS, PNLIPRP1, PNLIPRP2, CD36, } \\
\text { PLA2G1B }\end{array}$ \\
\hline hsa04610 & $\begin{array}{l}\text { Complement and coagulation } \\
\text { cascades }\end{array}$ & 6 & $1.0 \times 10^{-3}$ & F11, KLKB1, SERPINA5, C6, C5, F8 \\
\hline hsa00982 & $\begin{array}{l}\text { Drug metabolism- } \\
\text { cytochrome P450 }\end{array}$ & 5 & $7.1 \times 10^{-3}$ & GSTA1, GSTA2, AOX1, UGT2B11, ADH1B \\
\hline hsa00561 & Glycerolipid metabolism & 4 & $2.7 \times 10^{-2}$ & PNLIP, CEL, PNLIPRP1, PNLIPRP2 \\
\hline hsa04950 & $\begin{array}{l}\text { Maturity onset diabetes of the } \\
\text { young }\end{array}$ & 3 & $3.4 \times 10^{-2}$ & ONECUT1, IAPP, NR5A2 \\
\hline hsa00830 & Retinol metabolism & 4 & $3.7 \times 10^{-2}$ & ALDH1A1, AOX1, UGT2B11, ADH1B \\
\hline hsa04971 & Gastric acid secretion & 4 & $4.9 \times 10^{-2}$ & KCNJ16, CHRM3, CFTR, SST \\
\hline hsa00980 & $\begin{array}{l}\text { Metabolism of xenobiotics by } \\
\text { cytochrome P } 450\end{array}$ & 4 & $5.1 \times 10^{-2}$ & GSTA1, GSTA2, UGT2B11, ADH1B \\
\hline
\end{tabular}

B, Downregulated

\begin{tabular}{|c|c|c|c|c|}
\hline Pathway & Name & Count & P-value & Genes \\
\hline hsa04512 & ECM-receptor interaction & 17 & $1.4 \times 10^{-13}$ & $\begin{array}{l}\text { COL3A1, ITGB4, ITGA11, ITGA2, ITGA3, COL5A2, } \\
\text { LAMB3, LAMA3, COMP, ITGB6, COL6A3, COL1A2, } \\
\text { LAMC2, COL1A1, THBS2, COL11A1, FN1 }\end{array}$ \\
\hline hsa04510 & Focal adhesion & 18 & $1.1 \times 10^{-8}$ & $\begin{array}{l}\text { COL3A1, MET, ITGB4, ITGA11, ITGA2, ITGA3, COL5A2, } \\
\text { LAMB3, LAMA3, COMP, COL6A3, ITGB6, COL1A2, } \\
\text { LAMC2, COL1A1, THBS2, COL11A1, FN1 }\end{array}$ \\
\hline hsa04151 & PI3K-Akt signaling pathway & 21 & $20 \times 10^{-7}$ & $\begin{array}{l}\text { COL3A1, MET, ITGA11, ITGB4, ITGA2,ITGA3, COL5A2, } \\
\text { LAMB3, LAMA3, COMP, COL6A3, ITGB6, COL1A2, } \\
\text { LAMC2, EFNA5, IL2RG, COL1A1, THBS2, ANGPT2, } \\
\text { COL11A1, FN1 }\end{array}$ \\
\hline hsa05146 & Amoebiasis & 11 & $4.4 \times 10^{-6}$ & $\begin{array}{l}\text { IL1R2, LAMB3, LAMA3, COL3A1, COL1A2, LAMC2, } \\
\text { COL1A1, SERPINB3, COL11A1, COL5A2, FN1 }\end{array}$ \\
\hline hsa04974 & $\begin{array}{l}\text { Protein digestion and } \\
\text { absorption }\end{array}$ & 10 & $7.0 \times 10^{-6}$ & $\begin{array}{l}\text { KCNN4, COL17A1, COL3A1, COL6A3, COL1A2, } \\
\text { COL12A1, COL1A1, COL11A1, COL5A2, COL10A1 }\end{array}$ \\
\hline hsa05412 & $\begin{array}{l}\text { Arrhythmogenic right } \\
\text { ventricular cardiomyopathy }\end{array}$ & 7 & $7.2 \times 10^{-4}$ & DSG2, ITGB6, ITGA11, ITGB4, LEF1, ITGA2, ITGA3 \\
\hline hsa05202 & $\begin{array}{l}\text { Transcriptional misregulation } \\
\text { in cancer }\end{array}$ & 10 & $1.0 \times 10^{-3}$ & $\begin{array}{l}\text { PLAT, IL1R2, MMP9, MET, ETV1, RUNX2, HPGD, } \\
\text { HIST1H3H, PLAU, HIST1H3I }\end{array}$ \\
\hline hsa05222 & Small cell lung cancer & 6 & $9.6 \times 10^{-3}$ & LAMB3, LAMA3, ITGA2, LAMC2, ITGA3, FN1 \\
\hline hsa05230 & $\begin{array}{l}\text { Central carbon metabolism in } \\
\text { cancer }\end{array}$ & 5 & $1.6 \times 10^{-2}$ & SLC16A3, PKM, SLC2A1, MET, HK2 \\
\hline hsa05410 & Hypertrophic cardiomyopathy & 5 & $3.1 \times 10^{-2}$ & ITGB6, ITGA11, ITGB4, ITGA2, ITGA3 \\
\hline
\end{tabular}

KEGG, Kyoto Encyclopedia of Genes and Genomes; DEGs, differentially expressed genes; PDAC, pancreatic ductal adenocarcinoma. 


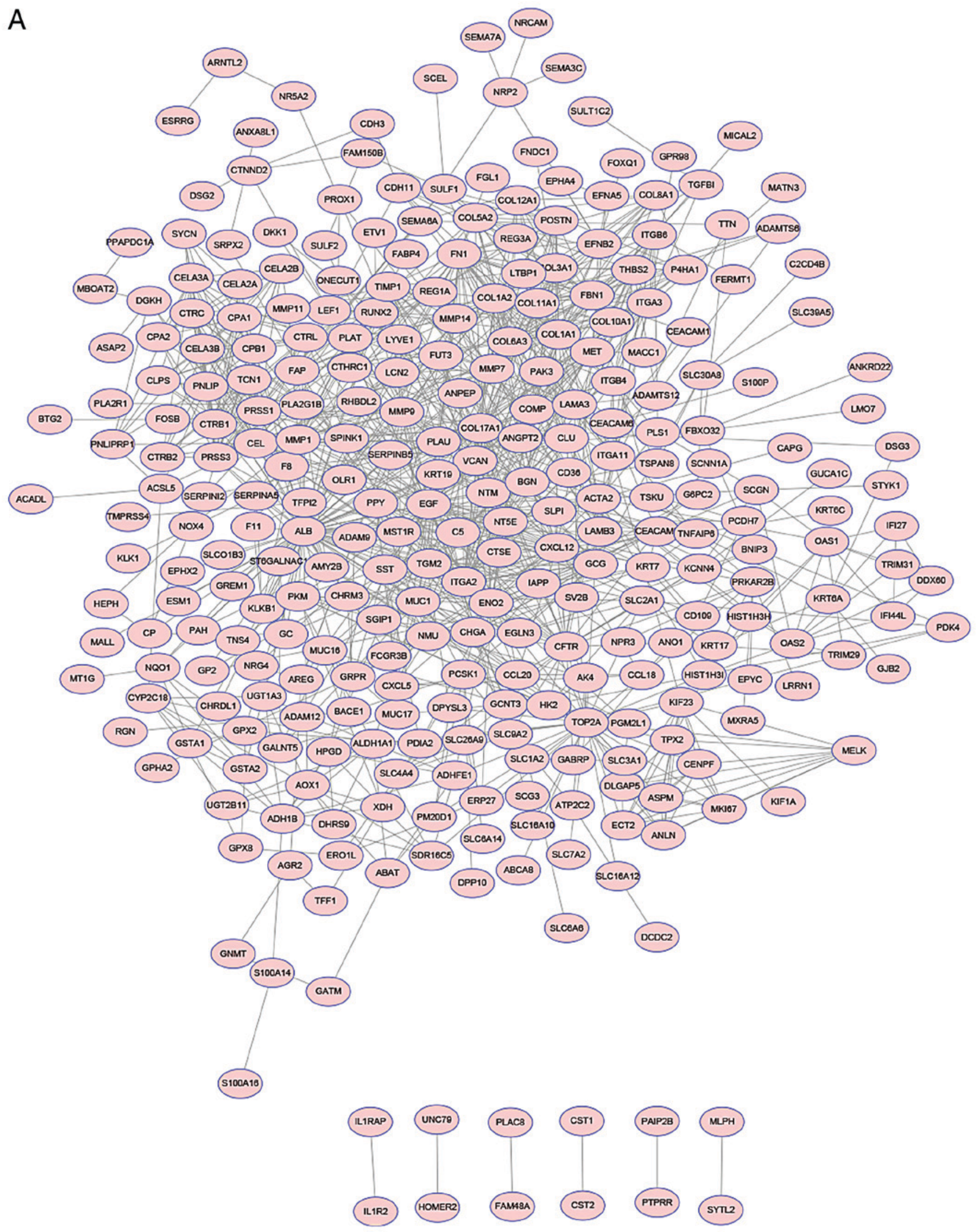

Figure 2. DEGs PPI network complex in PDAC. (A) All 386 DEG interaction networks.

thereby promoting cell carcinogenesis (27). Furthermore, the PI3K-Akt signaling pathway is important in the etiology of pancreatic cancer (28). Therefore, these signaling pathways can promote the development of pancreatic cancer in a variety of ways, and may provide a new direction for the systematic treatment of pancreatic cancer. 

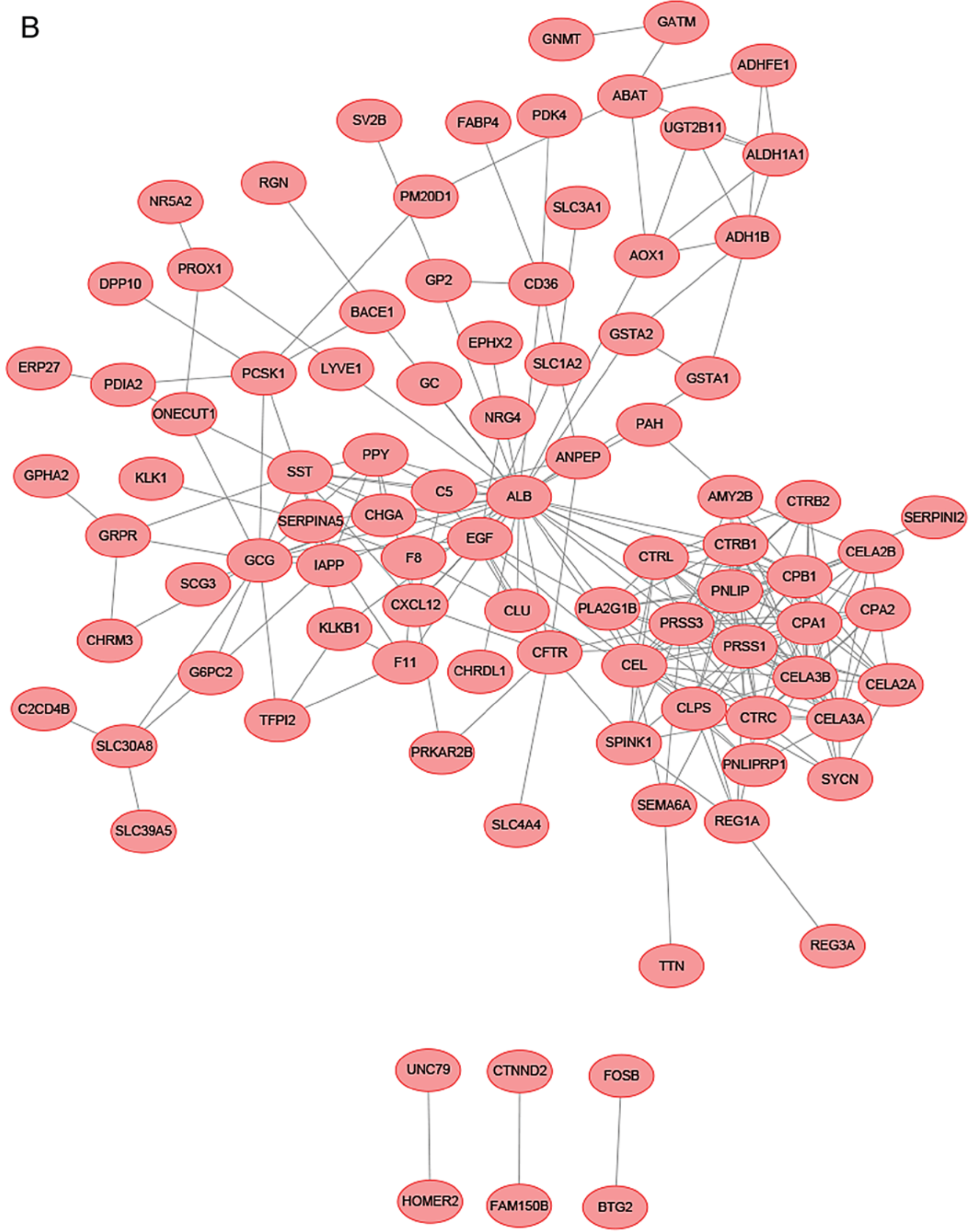

Figure 2. Continued. (B) The upregulated DEG interaction network.

In the current study, the top 10 degree hub genes identified in the PPI network were: CFTR, SLC7A2, CCL18,
PDK4, BAIAP2L1, ITGA3, CPA1, GPRC5A, STYK1 and ST6GALNAC1. CFTR was the highest scoring gene. The 


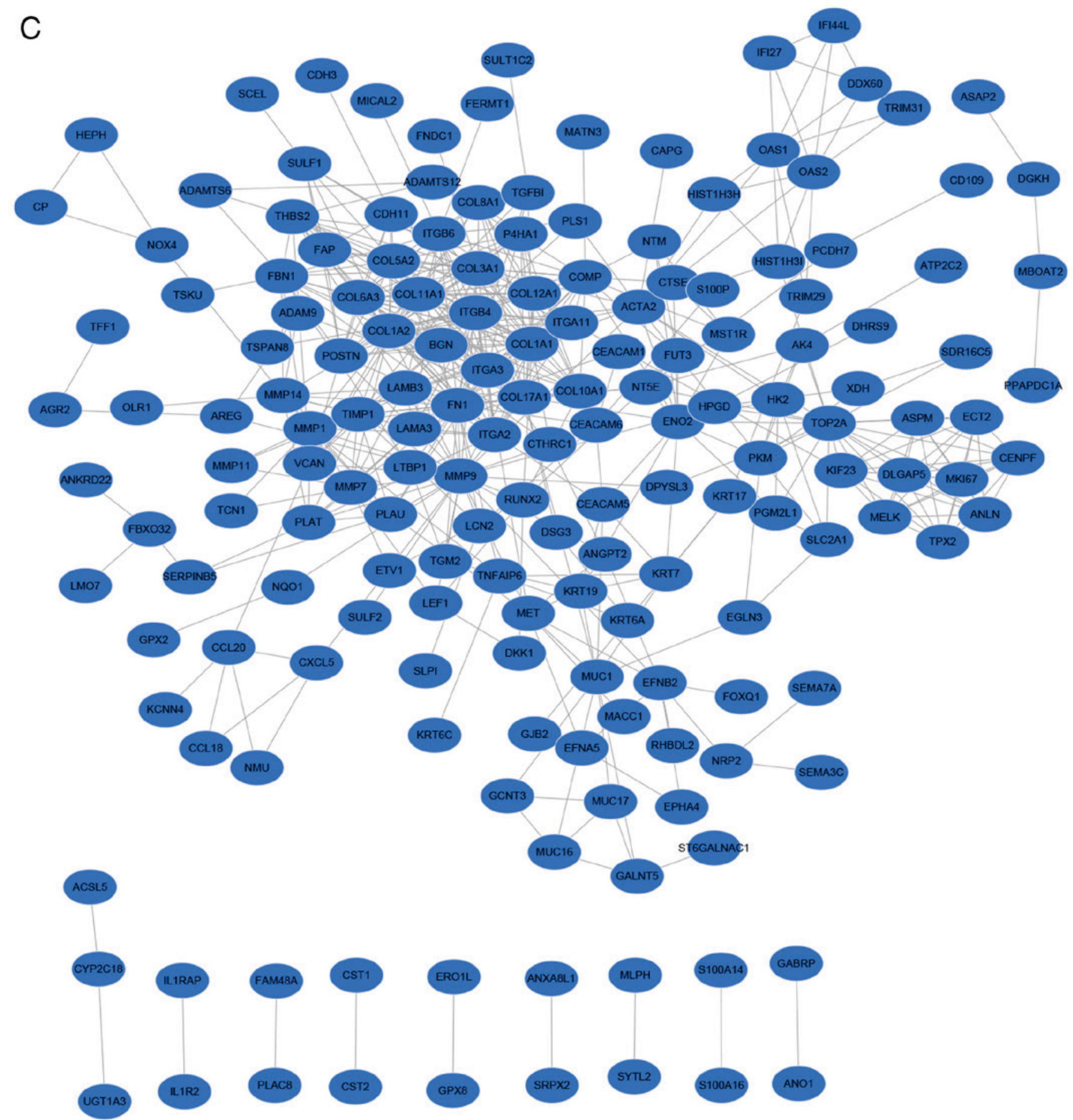

Figure 2. Continued. (C) The downregulated DEG interaction network. DEGs, differentially expressed genes; PPI, protein-protein interaction; PDAC, pancreatic ductal adenocarcinoma.

CFTR gene codes for the cystic fibrosis transmembrane conductance regulator protein, an important member of the ATP binding cassette transporter family (29). It serves an important role in anion regulation and tissue homeostasis of various epithelial cells, activates the cAMP channel and promotes chloride and bicarbonate secretion in the digestive system $(30,31)$. A previous study revealed that increased expression of CFTR in drug-resistant prostate cancer tissues or cells that block CFTR can inhibit tumor cell viability and autophagy via the PI3K/Akt signaling pathway (32).
In CFTR knockout mice, mucosal barrier function was impaired, including tight junction disruption, which resulted in impaired tolerance to bacterial colonization and infection, abnormal innate and adaptive immune responses, and inflammation $(33,34)$. It has been reported that CFTR is a negative regulator of the pro-inflammatory nuclear factor k-light-chain-enhancer of activated B cells-mediated innate immune response, including interleukin-8, and evokes a positive feedback loop of cyclooxygenase 2-prostaglandin E2 in inflammation, and therefore, these factors may work 
Table V. The enriched pathways of upregulated DEGs PPI network in PDAC.

A, Biological process

\begin{tabular}{llrr}
\hline Term & \multicolumn{1}{c}{ Description } & Count & P-value \\
\hline GO.0007586 & Digestion & 13 & $1.12 \times 10^{-8}$ \\
GO.0065008 & Regulation of biological quality & 45 & $7.78 \times 10^{-5}$ \\
GO.0044281 & Small molecule metabolic process & 36 & $1.85 \times 10^{-4}$ \\
GO.0046903 & Secretion & 18 & $1.85 \times 10^{-4}$ \\
GO.0002576 & Platelet degranulation & $7.08 \times 10^{-3}$ \\
\hline
\end{tabular}

B, Molecular function

\begin{tabular}{llll}
\hline Term & \multicolumn{1}{c}{ Description } & Count & P-value \\
\hline GO.0008236 & Serine-type peptidase activity & 13 & $2.61 \times 10^{-8}$ \\
GO.0004252 & Serine-type endopeptidase activity & 12 & $2.83 \times 10^{-7}$ \\
GO.0070011 & Peptidase activity, acting on L-amino acid peptides & 18 & $1.40 \times 10^{-5}$ \\
GO.0008233 & Peptidase activity & 18 & $1.77 \times 10^{-5}$ \\
GO.0004175 & Endopeptidase activity & 13 & $3.51 \times 10^{-3}$ \\
\hline
\end{tabular}

C, Cellular component

\begin{tabular}{|c|c|c|c|}
\hline Term & Description & Count & P-value \\
\hline GO.0005576 & Extracellular region & 76 & $2.57 \times 10^{-15}$ \\
\hline GO.0031988 & Membrane-bounded vesicle & 62 & $3.76 \times 10^{-12}$ \\
\hline GO.0044421 & Extracellular region part & 62 & $4.12 \times 10^{-11}$ \\
\hline GO.0005615 & Extracellular space & 35 & $8.18 \times 10^{-11}$ \\
\hline GO.0070062 & Extracellular exosome & 50 & $2.10 \times 10^{-9}$ \\
\hline
\end{tabular}

DEGs, differentially expressed genes; PPI, protein-protein interactions; PDAC, pancreatic ductal adenocarcinoma.

together to promote tumorigenesis $(35,36)$. The pancreas is a digestive organ that secretes a variety of substances to regulate the digestive fluids through exocrine and endocrine methods (37). At the same time, the abovementioned 10 hub genes can also regulate the development and progression of pancreatic cancer by regulating immune and inflammatory processes, protein glycosylation and energy metabolism which affect multiple signaling pathways (38-43). Therefore, these genes can be an important target for the precise treatment of pancreatic cancer.

For the upregulated DEGs, module analysis of the PPI network revealed that they were associated with pancreatic secretion signaling pathways and 'protein digestion and absorption' and 'lipid digestion and absorption' signaling pathways. Stimulation of the pancreas by secretagogues, including acetylcholine and cholecystokinin, results in intracellular $\mathrm{Ca}^{2+}$ signals, leading to the polarized secretion of enzymes (44). However, activation of the $\mathrm{CFTR}^{-}$channel and the CFTR-dependent $\mathrm{Cl}^{-} / \mathrm{HCO}_{3}{ }^{-}$exchange is responsible for cAMP-induced $\mathrm{HCO}_{3}{ }^{-}$secretion (44). The secretory function of the pancreas is directly associated with both protein and lipid metabolism in the body, the disruption of which may lead to chronic inflammation of the pancreas, developing into pancreatic cancer (45).

The downregulated DEGs were associated with ECM-receptor interactions, focal adhesion and the PI3K-Akt signaling pathway (46). The ECM serves an important role in the morphogenesis of tissues and organs, and in the maintenance of cell and tissue structures and functions (47). These interactions lead to direct or indirect control of cell activity, including adhesion, migration, differentiation, proliferation, and apoptosis (48). Furthermore, the focal adhesion signaling pathway is the key signaling pathway of cell matrix adhesion, which serves an important role in cell movement, cell proliferation, cell differentiation, gene expression regulation and cell survival (49). The proliferation and metastasis of cancer cells depend on the regulation of this pathway $(50,51)$. The PI3K-Akt signaling pathway serves as a bridge between extracellular signals and intracellular responses $(52,53)$. Once activated, Akt phosphorylation can be involved in apoptosis, matrix control, important cellular processes, protein synthesis, metabolism and the cell cycle (54). The results obtained in the 
Table VI. The enriched pathways of downregulated DEGs PPI network in PDAC.

A, Biological process

\begin{tabular}{llrr}
\hline Term & \multicolumn{1}{c}{ Description } & Count & P-value \\
\hline GO.0030198 & Extracellular matrix organization & 35 & $4.14 \times 10^{-24}$ \\
GO.0022617 & Extracellular matrix disassembly & 20 & $1.55 \times 10^{-17}$ \\
GO.0030574 & Collagen catabolic process & 15 & $6.65 \times 10^{-14}$ \\
GO.0007155 & Cell adhesion & 32 & $4.45 \times 10^{-9}$ \\
GO.0001704 & Formation of primary germ layer & 13 & $5.25 \times 10^{-9}$ \\
\hline
\end{tabular}

B, Molecular function

\begin{tabular}{llrr}
\hline Term & \multicolumn{1}{c}{ Description } & Count & P-value \\
\hline GO.0005201 & Extracellular matrix structural constituent & 10 & $5.72 \times 10^{-7}$ \\
GO.0005518 & Collagen binding & 8 & $4.92 \times 10^{-6}$ \\
GO.0005539 & Glycosaminoglycan binding & 13 & $1.12 \times 10^{-5}$ \\
GO.0005515 & Protein binding & 66 & $1.16 \times 10^{-4}$ \\
GO.0004222 & Metalloendopeptidase activity & 9 & $1.94 \times 10^{-4}$ \\
\hline
\end{tabular}

C, Cellular component

\begin{tabular}{llrr}
\hline Term & \multicolumn{1}{c}{ Description } & Count & P-value \\
\hline GO.0005576 & Extracellular region & 100 & $7.19 \times 10^{-25}$ \\
GO.0044421 & Extracellular region part & 91 & $2.70 \times 10^{-24}$ \\
GO.0005615 & Extracellular space & 44 & $1.93 \times 10^{-14}$ \\
GO.0070062 & Extracellular exosome & 63 & $4.76 \times 10^{-13}$ \\
GO.0031012 & Extracellular matrix & 24 & $6.44 \times 10^{-13}$
\end{tabular}

DEGs, differentially expressed genes; PPI, protein-protein interactions; PDAC, pancreatic ductal adenocarcinoma.

current study suggest that pancreatic secretory dysfunction, the imbalance of ECM-associated signaling pathways and the PI3K-Akt signaling pathway may result in cell cycle disruption and metabolism-associated microenvironmental changes, which can trigger the development of pancreatic cancer.

In conclusion, the current study investigated the biological pathways involved in PDAC by providing a comprehensive bioinformatics map of DEGs. These DEGs are involved in the development and progression of PDAC and provide a basis for the effective study of the molecular mechanisms of pancreatic cancer. Further molecular biological experiments and animal studies are required to confirm the functions and roles of these DEGs in PDAC.

\section{Acknowledgements}

Not applicable.

\section{Funding}

This study was supported by the Key Foundation of Sichuan MunicipalCommission of Health andFamily Planning Foundation of China (grant no. 17ZD008) and the Sichuan Medical Research Project Foundation of China (grant no. S16007).

\section{Availability of data and materials}

The datasets analyzed during the current study are available in the GSE28735 repository (www.ncbi.nlm.nih.gov/geo).

\section{Authors' contributions}

YH, YL and HW conceived of and designed the experiments. YH, YL, JG, CL and HZ performed the experiments. YH, YL and $\mathrm{HW}$ acquired, analyzed and interpreted the data and wrote the paper.

\section{Ethics approval and consent to participate}

Not applicable.

\section{Patient consent for publication}

Not applicable. 


\section{Competing interests}

The authors declare that they have no competing interests.

\section{References}

1. Ryan DP, Hong TS and Bardeesy N: Pancreatic adenocarcinoma. N Engl J Med 371: 2140-2141, 2014.

2. Quaresma M, Coleman MP and Rachet B: 40-year trends in an index of survival for all cancers combined and survival adjusted for age and sex for each cancer in England and Wales, 1971-2011: A population-based study. Lancet 385: 1206-1218, 2015.

3. Siegel R, Naishadham D and Jemal A: Cancer statistics, 2013. CA Cancer J Clin 63: 11-30, 2013.

4. Maron R, Schechter B, Mancini M, Mahlknecht G, Yarden Y and Sela M: Inhibition of pancreatic carcinoma by homo- and heterocombinations of antibodies against EGF-receptor and its kin HER2/ErbB-2. Proc Natl Acad Sci USA 110: 15389-15394, 2013.

5. Chen YL, Hu CM, Hsu JT, Chang CC, Huang TY, Chiang PH, Chen WY, Chang YT, Chang MC, Tien YW, et al: Cellular 5-hydroxylmethylcytosine content determines tumorigenic potential and prognosis of pancreatic ductal adenocarcinoma Am J Cancer Res 8: 2548-2563, 2018.

6. Capello M, Vykoukal JV, Katayama H, Bantis LE, Wang H, Kundnani DL, Aguilar-Bonavides C, Aguilar M, Tripathi SC, Dhillon DS, et al: Exosomes harbor B cell targets in pancreatic adenocarcinoma and exert decoy function against complement-mediated cytotoxicity. Nat Commun 10: 254, 2019.

7. Chu LC, Goggins MG and Fishman EK: Diagnosis and detection of pancreatic cancer. Cancer J 23: 333-342, 2017.

8. Ilic $\mathrm{M}$ and Ilic I: Epidemiology of pancreatic cancer. World $\mathrm{J}$ Gastroenterol 22: 9694-9705, 2016.

9. Vogelstein B, Papadopoulos N, Velculescu VE, Zhou S, Diaz LA Jr and Kinzler KW: Cancer genome landscapes. Science 339: 1546-1558, 2013.

10. Lin QJ, Yang F, Jin C and Fu DL: Current status and progress of pancreatic cancer in China. World J Gastroenterol 21: 7988-8003, 2015.

11. Yan X, Wan H, Hao X, Lan T, Li W, Xu L, Yuan K and Wu H: Importance of gene expression signatures in pancreatic cancer prognosis and the establishment of a prediction model. Cancer Manag Res 11: 273-283, 2018.

12. Zhang G, Schetter A, He P, Funamizu N, Gaedcke J, Ghadimi BM, Ried T, Hassan R, Yfantis HG, Lee DH, et al: DPEP1 inhibits tumor cell invasiveness, enhances chemosensitivity and predicts clinical outcome in pancreatic ductal adenocarcinoma. PLoS One 7: e31507, 2012

13. Zhou S, Liu P, Jiang $W$ and Zhang H: Identification of potential target genes associated with the effect of propranolol on angiosarcoma via microarray analysis. Oncol Lett 13: 4267-4275, 2017.

14. Huang $\mathrm{H}$ and Brekken RA: The next wave of stroma-targeting therapy in pancreatic cancer. Cancer Res 79: 328-330, 2019.

15. Siegel RL, Miller KD and Jemal A: Cancer statistics, 2016. CA Cancer J Clin 66: 7-30, 2016.

16. Heinemann V, Reni M, Ychou M, Richel DJ, Macarulla T and Ducreux M: Tumour-stroma interactions in pancreatic ductal adenocarcinoma: Rationale and current evidence for new therapeutic strategies. Cancer Treat Rev 40: 118-128, 2014.

17. Zhang G, He P, Tan H, Budhu A, Gaedcke J, Ghadimi BM Ried T, Yfantis HG, Lee DH, Maitra A, et al: Integration of metabolomics and transcriptomics revealed a fatty acid network exerting growth inhibitory effects in human pancreatic cancer. Clin Cancer Res 19: 4983-4993, 2013.

18. Zhang G, Schetter A, He P, Funamizu N, Gaedcke J, Ghadimi BM, Ried T, Hassan R, Yfantis HG, Lee DH, et al: DPEP1 inhibits tumor cell invasiveness, enhances chemosensitivity and predicts clinical outcome in pancreatic ductal adenocarcinoma. PLoS One 7: e31507, 2012.

19. Ling YH, Ren CH, Guo XF, Xu LN, Huang YF, Luo JC, Zhang YH, Zhang XR and Zhang ZJ: Identification and characterization of microRNAs in the ovaries of multiple and uniparous goats (Capra hircus) during follicular phase. BMC Genomics 15: 339,2014

20. Raimondi S,Lowenfels AB, Morselli-Labate AM, Maisonneuve P and Pezzilli R: Pancreatic cancer in chronic pancreatitis; aetiology, incidence, and early detection. Best Pract Res Clin Gastroenterol 24: 349-358, 2010
21. Grippo PJ and Munshi HG: Imaging the Pancreatic ECM. Pancreatic Cancer and Tumor Microenvironment. Trivandrum (India): Transworld Research Network: Chapter 2, 2012.

22. Hanahan D and Weinberg RA: Hallmarks of cancer: The next generation. Cell 144: 646-674, 2011

23. Le A, Rajeshkumar NV, Maitra A and Dang CV: Conceptual framework for cutting the pancreatic cancer fuel supply. Clin Cancer Res 18: 4285-4290, 2012.

24. Apte MV, Pirola RC and Wilson JS: Pancreatic stellate cells: A starring role in normal and diseased pancreas. Front Physiol 3: $344,2012$.

25. Koikawa K, Ohuchida K, Takesue S, Ando Y, Kibe S, Nakayama H, Endo S, Abe T, Okumura T, Horioka K, et al: Pancreatic stellate cells reorganize matrix components and lead pancreatic cancer invasion via the function of Endo180. Cancer Lett 412: 143-154, 2018

26. Grzesiak JJ, Ho JC, Moossa AR and Bouvet M: The integrin-extracellular matrix axis in pancreatic cancer. Pancreas 35 : 293-301, 2007.

27. Burridge K: Focal Adhesions: A personal perspective on a half century of progress. FEBS J 284: 3355-3361, 2017.

28. Ebrahimi S, Hosseini M, Shahidsales S, Maftouh M, Ferns GA, Ghayour-Mobarhan M, Hassanian SM and Avan A: Targeting the Akt/PI3K signaling pathway as a potential therapeutic strategy for the treatment of pancreatic cancer. Curr Med Chem 24: 1321-1331, 2017.

29. Zou WB, Tang XY, Zhou DZ, Qian YY, Hu LH, Yu FF, Yu D, Wu H, Deng SJ, Lin JH, et al: SPINK1, PRSS1, CTRC, and CFTR genotypes influence disease onset and clinical outcomes in chronic pancreatitis. Clin Transl Gastroenterol 9: 204, 2018.

30. Collins FS: Cystic fibrosis: Molecular biology and therapeutic implications. Science 256: 774-779, 1992.

31. Anderson MP, Gregory RJ, Thompson S, Souza DW, Paul S, Mulligan RC, Smith AE and Welsh MJ: Demonstration that CFTR is a chloride channel by alteration of its anion selectivity. Science 253: 202-205, 1991

32. Zhu Q, Li H, Liu Y and Jiang L: Knockdown of CFTR enhances sensitivity of prostate cancer cells to cisplatin via inhibition of autophagy. Neoplasma 64: 709-717, 2017.

33. De Lisle RC: Disrupted tight junctions in the small intestine of cystic fibrosis mice. Cell Tissue Res 355: 131-142, 2014.

34. Munck A: Cystic fibrosis: Evidence for gut inflammation. Int $\mathrm{J}$ Biochem Cell Biol 52: 180-183, 2014.

35. Vij N, Mazur S and Zeitlin PL: CFTR is a negative regulator of NFkappaB mediated innate immune response. PLoS One 4: e4664, 2009.

36. Chen J, Jiang XH, Chen H, Guo JH, Tsang LL, Yu MK, $\mathrm{Xu}$ WM and Chan HC: CFTR negatively regulates cyclooxygenase-2-PGE(2) positive feedback loop in inflammation. J Cell Physiol 227: 2759-2766, 2012.

37. Brereton MF, Iberl M, ShimomuraK,Zhang Q, Adriaenssens AE, Proks P, Spiliotis II, Dace W, Mattis KK, Ramracheya R, et al: Reversible changes in pancreatic islet structure and function produced by elevated blood glucose. Nat Commun 5: 4639, 2014.

38. Sun P, Zhu X, Shrubsole MJ, Ness RM, Hibler EA, Cai Q, Long J, Chen Z, Li G, Hou L, et al: Genetic variation in SLC7A2 interacts with calcium and magnesium intakes in modulating the risk of colorectal polyps. J Nutr Biochem 47: 35-40, 2017.

39. Chen J, Yao Y, Gong C, Yu F, Su S, Chen J, Liu B, Deng H, Wang F, Lin L, et al: CCL18 from tumor-associated macrophages promotes breast cancer metastasis via PITPNM3. Cancer Cell 19: 541-555, 2011.

40. Bonnet S, Archer SL, Allalunis-Turner J, Haromy A, Beaulieu C, Thompson R, Lee CT, Lopaschuk GD, Puttagunta L, Bonnet $\mathrm{S}$, et al: A mitochondria- $\mathrm{K}+$ channel axis is suppressed in cancer and its normalization promotes apoptosis and inhibits cancer growth. Cancer Cell 11: 37-51, 2007.

41. Wang YP, Huang LY, Sun WM, Zhang ZZ, Fang JZ, Wei BF, $\mathrm{Wu} \mathrm{BH}$ and Han ZG: Insulin receptor tyrosine kinase substrate activates EGFR/ERK signalling pathway and promotes cell proliferation of hepatocellular carcinoma. Cancer Lett 337: 96-106, 2013.

42. Yang L, Ma T and Zhang J: GPRC5A exerts its tumor-suppressive effects in breast cancer cells by inhibiting EGFR and its downstream pathway. Oncol Rep 36: 2983-2990, 2016.

43. Wang J, Farris AB, Xu K, Wang P, Zhang X, Duong DM, Yi H, Shu HK, Sun SY and Wang Y: GPRC5A suppresses protein synthesis at the endoplasmic reticulum to prevent radiation-induced lung tumorigenesis. Nat Commun 7: 11795, 2016. 
44. Chey WY and Chang T: Neural hormonal regulation of exocrine pancreatic secretion. Pancreatology 1: 320-335, 2001.

45. Vaziri-Gohar A, Zarei M, Brody JR and Winter JM: Metabolic dependencies in pancreatic cancer. Front Oncol 8: 617, 2018.

46. Rijkers AP, Bakker OJ, Ahmed Ali U, Hagenaars JCJP, van Santvoort HC, Besselink MG, Bollen TL and van Eijck CH; Dutch Pancreatitis Study Group: Risk of pancreatic cancer after a primary episode of acute pancreatitis. Pancreas 46: 1018-1022, 2017.

47. Theocharis AD, Skandalis SS, Gialeli C and Karamanos NK: Extracellular matrix structure. Adv Drug Deliv Rev 97: 4-27, 2016.

48. Canobbio I, Balduini C and Torti M: Signalling through the platelet glycoprotein Ib-V-IX complex. Cell Signal 16: 1329-1344, 2004.

49. Eke I and Cordes N: Focal adhesion signaling and therapy resistance in cancer. Semin Cancer Biol 31: 65-75, 2015.
50. Guo W and Giancotti FG: Integrin signalling during tumour progression. Nat Rev Mol Cell Biol 5: 816-826, 2004.

51. Lee JW and Juliano R: Mitogenic signal transduction by integrin- and growth factor receptor-mediated pathways. Mol Cells 17: 188-202, 2004

52. Engelman JA, Luo J and Cantley LC: The evolution of phosphatidylinositol 3-kinases as regulators of growth and metabolism. Nat Rev Genet 7: 606-619, 2006.

53. Song G, Ouyang G and Bao S: The activation of Akt/PKB signaling pathway and cell survival. J Cell Mol Med 9: 59-71, 2005.

54. Hers I, Vincent EE and Tavaré JM: Akt signalling in health and disease. Cell Signal 23: 1515-1527, 2011.

(c) (i) This work is licensed under a Creative Commons Attribution 4.0 International (CC BY 4.0) License. 